\title{
Primary User Channel State Prediction Based on Markov Switching Model
}

\author{
Ahmed Mohammed Mikaeil, Bin Guo \& Xuemei Bai \\ School of Electronics and Information Engineering, Changchun University of Science and Technology, \\ Changchun, Jilin Province, China \\ Zhijun Wang \\ Jilin Engineering Research Center of RFID and Intelligent Information Processing, Jilin, Changchun, China
}

\begin{abstract}
The most important challenge of the spectrum sensing is to find a way to share the licensed spectrum without interfering with the licensed users transmission. Therefore, predicting the licensed or primary users (PU) channel occupancy status has been investigated extensively in recent years, this study introduce a novel approach for predicting the PU channel state based on Markov switching model. In this approach we model the detected primary user channel state, which can be represented by two states; PU channel "idle" or "occupied" as a time series changing "switching" over the time between two Gaussian distribution according to the detection sequence. Then we fed this time series into the Markov switching model to predict these switching "changes" before they happen so that the secondary user (SU) can adjust their transmission strategies accordingly. The experimental results show the efficiency of the new approach for predicting the PU channel occupancy status.
\end{abstract}

KEYWORD: Channel state prediction; Channel occupancy status; Markov switching model; Maximum likelihood estimation; Primary users

\section{INTRODUCTION}

In spectrum sensing for cognitive radio network it is very important for the secondary user to be able predict the PU channel state in the near future using previous detected channel states in order to be ready to occupy or leave the channel for the PU when needed to prevent the harmful interference with primary users. The prediction of PU channel state has been studied in many literatures recently. For examples, the idea of predictive dynamic spectrum access has been introduced in (Clancy, T., \& Walker, B. 2006). The binary time series approach for spectrum occupancy characterization and prediction has been proposed in (Yarkan \& Arslan 2007). The channel state prediction using hidden Markov model (HMM) based on pattern recognition has been studied in (Park et al 2007). The prediction of channel state based on HMM and modified HMM has been introduced in (Chen \& Qiu, 2011. Channel) and (Chen \& Qiu 2010). An approach of single secondary user prediction based on modified HMM and soft-combining decision rule for primary uses prediction has been proposed in (Chen \& Qiu 2011. Experimental). However, all of these previous works have focused on HMM model approach for predicting the PU channel state, while the nature of the PU channel state is a two states "idle " or " busy "switching process over the time. Furthermore, it has shown in many other applications in practical areas such as bioinformatics, biology, economics, finance, marketing, medicine, and speech recognition that such a switching process can be predicted accurately using Markov switching process. Therefore, we introduce a novel approach for predicting PU channel state " occupancy status " based on Markov switching model.

The paper is organized as follows: the system model for the energy detection and generating the time series for Markov switching model is presented in section 2. In Section 3, we model the generated time series using two-states Markov model, followed by the estimation of the Markov switching model parameters using maximum in section 4 . The simulation results are presented in section 5. Section 6 concludes this article1.

\section{SYSTEM MODEL}

In this study we model the system of predicting the primary user channel occupancy in two model; first detecting the primary user channel state (PU signal present or absent) using the traditional single user energy detector "data fusion or any others spectrum sensing approach could be used for this propose", 
then generating a time series that capture the detection sequence model. The block diagram in Figure 1 illustrates the system model used for the prediction.

\begin{tabular}{|c|c|c|}
\hline $\begin{array}{l}\mathrm{Y}_{\mathrm{t}} \\
=\frac{1}{\mathrm{~N}} \sum_{\mathrm{n}=1}^{\mathrm{N}} \mid\left(\mathrm{y}_{\mathrm{t}}(\mathrm{n})\right)\end{array}$ & $\begin{array}{c}\text { Time series } \\
z_{t}, \\
t=1, \ldots, T\end{array}$ & $\begin{array}{l}\text { Markov } \\
\text { Switched Model } \\
\mathrm{z}_{\mathrm{t}}, \mathrm{t}=1, \ldots, \mathrm{T} \\
\mathrm{s}_{\mathrm{t}}, \in(0,1) \text { State }\end{array}$ \\
\hline
\end{tabular}

Fig. 1. The block diagram of PU channel state perdition based on Markov Switching Model

\subsection{Energy detection model}

In energy detection based spectrum sensing the secondary user sensor observe the licensed spectrum to determine whether the primary user signal is present or absent on it by doing the binary hypothesis test on the received signal over a the time $1 \leq t \leq T$ as follow:

$$
y_{t}(n)=\left\{\begin{array}{cc}
w_{t}(n) & H 0(P U \text { absent }) \\
\sqrt{\gamma_{t}} s_{t}(n)+w_{t}(n) & H 1(P U \text { present })
\end{array}\right.
$$

where $y_{t}(n)$ the observed received signal, $s_{t}(n)$ the primary user's signal, and it is assumed to be Gaussian i.i.d random process with zero mean and variance $\sigma_{s}{ }^{2}, w_{i}(n)$ is the noise, and assumed to be Gaussian i.i.d random process with zero mean and variance $\sigma_{u}{ }^{2}, \gamma_{t}$ the SNR at time $t, N$ the number of samples used by SU sensing node and $T$ the overall system time. The instantaneous energy statistic for thetat the secondary user sensing node $Y_{t}$ can be represented by:

$$
Y_{t}=\frac{1}{N} \sum_{n=1}^{N}\left|\left(y_{t}(n)\right)\right|^{2} \quad, 1 \leq t \leq T
$$

where $Y_{\mathrm{t}}$ is a random variable whose probability density function (PDF) is chi-square distribution with $2 N$ degrees of freedom for complex value, and with $N$ degrees of freedom for real value case. If $(\mathrm{N} \geq 200) Y_{t}$ can be approximated by Gaussian distribution and the distribution of the power test $Y_{t}$ for wide band signal follows:

$$
Y_{t} \sim \begin{cases}\left(\sigma_{t}^{2}, 2 \sigma_{t}^{4} / N\right) & H 0 \\ \left(\sigma_{t}^{2}\left(1+\gamma_{t}\right), 2 \sigma_{t}^{4}\left(1+\gamma_{t}\right)^{2} / N\right. & H 1\end{cases}
$$

Under the assumption that the noise variance and the SNR stay the same during the observation time then $\sigma_{t}{ }^{2}=\sigma_{u}{ }^{2}, \gamma_{t}=\gamma_{u}$, and for a chosen threshold $\lambda_{t}=\lambda$ the probability of the false alarm $P_{f}$ can be written as:

$$
\begin{aligned}
P_{f}(\lambda)=\operatorname{Pr}\left(Y_{t}>\lambda \mid H 0\right) & =\frac{1}{\sqrt{2 \pi \sigma_{u}}} \int_{\lambda}^{\infty} e^{-\left(\lambda-\sigma_{u}\right)^{2} / \sqrt{2} \sigma_{u}{ }^{2}} \\
& =Q\left(\left(\frac{\lambda}{\sigma_{u}{ }^{2}}-1\right)\right)
\end{aligned}
$$

where $Q($.$) is the complementary distribution$ function of Gaussian distribution with zero mean and unit variance. From equation (4) and for a given probability of false alarm $P_{f}$, the single user threshold can be written as:

$$
\lambda=\left(\sqrt{\frac{2}{N}} Q^{-1}\left(P_{f}\right)+1\right) \sigma_{u}^{2}
$$

where $Q^{-1}($.$) is the inverse of theQ (.) function. And$ the decision of the sensing results over the time can be written as follow

$$
D_{t}=\left\{\begin{array}{ccc}
\text { "0" } & \text { PU absent } & Y_{t}<\lambda \\
" 1 " & \text { PU present } & Y_{t} \geq \lambda
\end{array} \quad 0 \leq t \leq T\right.
$$

\subsection{Time series generation model}

We assume that the PU channel state (absent, present) over the time has been already detected using "energy detector " as in subsection 2.1. If, we denote the period that the primary user stays absent or inactive as "idle state" and the period that the PU present or active as "occupied state". Our goal is to the predict the time when the channel state will going to switch between these two states, for example from "idle" to "occupied " and vice versa. For predicting this kind of switching process, we generate a time series $z_{t}$ to capture the detection sequence signal $D_{t}$ and assume that $z_{t}$ follow two different Gaussian distributions $N \sim\left(\mu_{\mathrm{z} 1}, \sigma_{\mathrm{z} 1}{ }^{2}\right)$ or $N \sim\left(\mu_{z 0}, \sigma_{z 0}^{2}\right)$ according to the detected PU channel state "PU present" or "absent" as follow:

$$
z_{t} \sim\left\{\begin{array}{cc}
\left(\mu_{z 0}, \sigma_{z 0}{ }^{2}\right) & Y_{t}<\lambda \\
\left(\mu_{z 1}, \sigma_{z 1}{ }^{2}\right) & Y_{t} \geq \lambda
\end{array} \quad 1 \leq t \leq T\right.
$$

Equation (8) formulates a two state Gaussian regime-switching time series. Markov switching model has been widely used in predicting the switching "change" in such a time series.

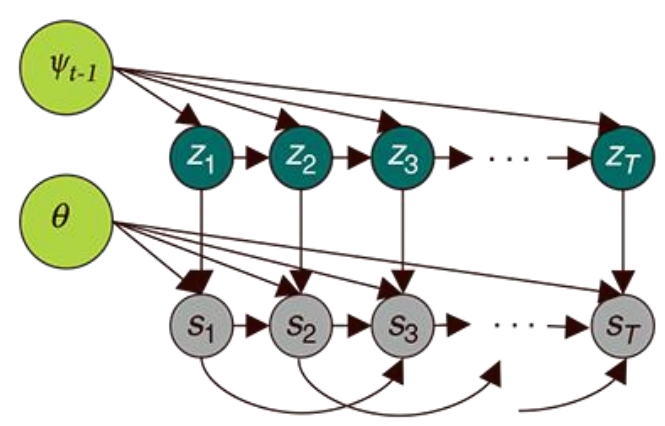

Fig. 2. Markov Switching Model

\section{MARKOV SWITCHING MODEL}

A simple Markov switching regression model for the time series $z_{t}$ can written as in (Frühwirth-Schnatter 2006) as:

$$
z_{t}=\mu_{s_{t}}+\epsilon_{t} \quad, \epsilon_{t} \sim\left(0, \sigma_{s_{t}}{ }^{2}\right)
$$

where $\mu_{s_{t}}$ is a row vector of predetermined explanatory variables measured at time $t$, which may 
include the lagged values of $z_{t}, \epsilon_{t}$ is the white noise process, $s_{t}=\{0,1\}$ is a hidden Markov chain with a regime-dependent mean equal to $\mu_{s t}=\mu_{0}(1-$ $\left.s_{t}\right)+\mu_{1} s_{t}$ and standard deviation $\sigma_{s t}=\sigma_{0}(1-$ $\left.s_{t}\right)+\sigma_{1} s_{t}$ over the time. The state variable $s_{t}$ follows a first order two-state Markov chain (Kuan 2002). Given the past history of $s_{t}$ the probability of $s_{t}$ takes certain value only depends on $s_{t-1}$, which take the Markov property:

$$
P\left(s_{t}=j \mid s_{t-1}=i\right)=P_{i j}
$$

where $P_{i j}(i ; j=0 ; 1)$ denote the transition probabilities of $s_{t}=j$ given that $s_{t-1}=i$. Clearly, the transition probabilities satisfy $P_{i 0}+P_{i 1}=1$. We often collect the transition probabilities $P_{i j}$ into a transition matrix as:

$$
\begin{aligned}
P & =\left(\begin{array}{ll}
P\left(s_{t}=0 \mid s_{t-1}=0\right) & P\left(s_{t}=0 \mid s_{t-1}=1\right) \\
P\left(s_{t}=1 \mid s_{t-1}=0\right) & P\left(s_{t}=1 \mid s_{t-1}=1\right)
\end{array}\right) \\
& =\left(\begin{array}{ll}
P_{00} & P_{01} \\
P_{10} & P_{11}
\end{array}\right)
\end{aligned}
$$

where $P$ is the transition matrix that governs the random behavior of the state variable $s_{t}$, and it contains only two parameters $\left(P_{00}\right.$ and $\left.P_{11}\right)$. Assuming that we do not observe $s_{t}$ directly, but only infer its operation through the observed behavior of $z_{t}$. The parameters necessary to fully describe the probability law governing $z_{t}$ are the variance of the Gaussian innovation $\sigma_{0}, \sigma_{1}$, the expectation of the dependent variable $\mu_{0}$ and $\mu_{1^{\prime}}$, and the two state transition probabilities, $P_{00}$ and $P_{11}$, all these parameters requires full estimation, via maximum likelihood estimation.

\section{MAXIMUM LIKELIHOOD ESTIMATION OF MARKOV SWITCHING MODEL}

There are various ways to estimate the parameters for the Markov switching model such as Quasimaximum likelihood estimation (QMLE), Gibbs sampling and etc. In this study we focus on maximum likelihood estimation (MLE). If we let $\psi_{t-1}=\left\{z_{t-1}, z_{t}, z_{t+1} \ldots z_{1}\right\}$ to be a vector of the available information until time $t-1, \theta=\left\{\sigma_{0}, \sigma_{1}\right.$, $\left.\mu_{0}, \mu_{1}, P_{00}, P_{11}\right\}$ to be the vector of Markov switching model parameters and $\psi_{L}=\left\{z_{t-1}, z_{t}, \ldots z_{L}\right\}$ to a vector information till $L$ sample see Fig. 2 , in order to assess the likelihood of the state variable $s_{t}$ based on what happening with $z_{t}$, we need to evaluate its optimal forecasts (conditional expectations) $s_{t}=i, \quad(i=0,1)$, based on $\psi$ and $\theta$. These forecasts include prediction probabilities $P\left(s_{t}=i \mid \psi_{t-1} ; \theta\right)$, which are based on the information prior to time $t$, filtering probabilities $P\left(s_{t}=i \mid \psi_{t} ; \theta\right)$ which are based on the past and current information, and smoothing probabilities $P\left(s_{t}=i \mid \psi_{L} ; \theta\right)$ which are based on the full-sample information. By deriving these probabilities, we can obtain the log-likelihood function as a byproduct, and then compute the maximum likelihood estimates.

Under the normality assumption, the density of $z_{t}$ conditional on $\psi_{t-1}$ and $s_{t}=i,(i=0,1)$ is given by:

$$
\mathcal{F}\left(z_{t} \mid s_{t}=i, \psi_{t-1} ; \theta\right)=\frac{1}{\sqrt{2 \pi \sigma_{s t}}} e^{-\frac{\left(z_{t}-\mu_{s}\right)^{2}}{2 \sigma_{s t}{ }^{2}}}
$$

where $\mathcal{F}$ denote the probability density function. Given the prediction probabilities $P\left(s_{t}=\right.$ $\left.i \mid \psi_{t-1} ; \theta\right)$ the density of $z_{t}$ conditional on $\psi_{t-1}$ alone can be obtained as:

$$
\begin{aligned}
& \mathcal{F}\left(z_{t} \mid \psi_{t-1} ; \theta\right) \\
& \quad=P\left(s_{t}=0 \mid \psi_{t-1} ; \theta\right) \mathcal{F}\left(z_{t} \mid s_{t}=0 \psi_{t-1} ; \theta\right) \\
& \quad+P\left(s_{t}=1 \mid \psi_{t-1} ; \theta\right) \mathcal{F}\left(z_{t} \mid s_{t}=1 \psi_{t-1} ; \theta\right)
\end{aligned}
$$

For $i=0 ; 1$, the filtering probabilities of $s_{t}$ are:

$$
\begin{aligned}
P\left(s_{t}\right. & \left.=i \mid \psi_{t} ; \theta\right) \\
& =\frac{P\left(s_{t}=i \mid \psi_{t-1} ; \theta\right) \mathcal{F}\left(z_{t} \mid s_{t}=i \psi_{t-1} ; \theta\right)}{\mathcal{F}\left(z_{t} \mid \psi_{t-1} ; \theta\right)}
\end{aligned}
$$

The prediction probabilities are:

$$
\begin{aligned}
P\left(s_{t+1}=\right. & \left.i \mid \psi_{t} ; \theta\right) \\
= & P\left(s_{t}=0, \mathrm{~s}_{\mathrm{t}+1}=i \mid \psi_{t} ; \theta\right) \\
& +P\left(s_{t}=1, \mathrm{~s}_{\mathrm{t}+1}=i \mid \psi_{t} ; \theta\right) \\
= & P_{0 i} P\left(s_{t}=0 \mid \psi_{t} ; \theta\right) \\
& +P_{1 i} P\left(s_{t}=1 \mid \psi_{t} ; \theta\right)
\end{aligned}
$$

where $P_{0 i}=P\left(s_{t+1}=i \mid s_{t}=0\right)$ and $P_{1 i}=P\left(s_{t+1}=\right.$ $\left.i \mid s_{t}=1\right) \quad$ are transition probabilities. By setting the initial values as given in (Hamilton, 1994) assuming the Markov chain is presumed to be ergodic:

$$
P\left(s_{0}=i \mid \psi_{0}\right)=\frac{1-P_{j j}}{2-P_{i i}-P_{j j}}
$$

we can iterate the equations (13) and (14) to obtain the filtering probabilities $P\left(s_{t+1}=i \mid s_{t}=0\right)$ as well as the conditional densities $\mathcal{F}\left(z_{t} \mid s_{t}=\right.$ $\left.0 \psi_{t-1} ; \theta\right)$ for $t=1,2, \ldots . T$. The log-likelihood function is therefore:

$$
\begin{aligned}
\log (L(\hat{\theta}))= & \sum_{t=1}^{T} \sum_{i=1}^{2} \log \left(\mathcal{F}\left(z_{t} \mid \psi_{t-1} ; \theta\right)\right. \\
& \left.\times P\left(S_{t}=i \mid \psi_{t} ; \theta\right)\right)
\end{aligned}
$$

where $L(\hat{\theta})$ is the maximized value of the likelihood function. The model estimation is obtained by finding the set of parameters $\hat{\theta}$ that maximize the previous equationby using for example numerical-search algorithm. The estimated filtering and prediction probabilities are then easily calculated by plugging $\hat{\theta}$ into the formulae for these probabilities. To compute the smoothing probabilities $P\left(s_{t}=i \mid \psi_{L} ; \theta\right)$, we adopt the approximation in (Kim 1994) and for $i ; j=$ $0 ; 1$, smoothing probabilities can be expressed as: 


$$
\begin{aligned}
& P\left(s_{t}=i 0 \mid \psi_{L} ; \theta\right) \\
&= P\left(s_{t+1}=0 \mid \psi_{L} ; \theta\right) P\left(s_{t}=i \mid s_{t+1}=0, \psi_{L} ; \theta\right) \\
&+P\left(s_{t+1}=1 \mid \psi_{L} ; \theta\right) P\left(s_{t}=i \mid s_{t+1}=0, \psi_{L} ; \theta\right. \\
&= P\left(s_{t}=i \mid \psi_{t} ; \theta\right) \\
& \quad \times\left(\frac{P_{i 0} P\left(s_{t+1}=0 \mid \psi_{L} ; \theta\right)}{P\left(s_{t+1}=0 \mid \psi_{t} ; \theta\right)}+\frac{P_{i 1} P\left(s_{t+1}=1 \mid \psi_{L} ; \theta\right.}{P\left(s_{t+1}=1 \mid \psi_{t} ; \theta\right)}\right)
\end{aligned}
$$

Using the filtering probability $P\left(S_{L}=i \mid \psi_{L} ; \theta\right)$ as the initial value, we can iterate backward the equations for filtering and prediction probabilities and the equation above to get the smoothing probabilities for $t=L-1, \cdots, 1$.

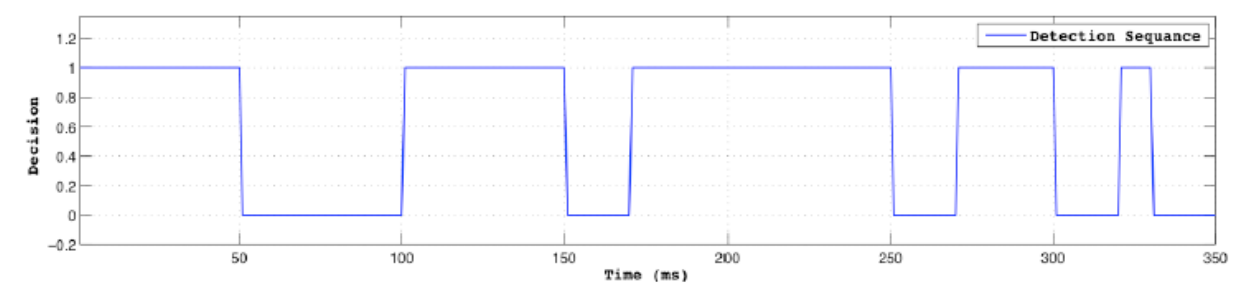

Fig. 3.Shows PU channel sensing detection sequence
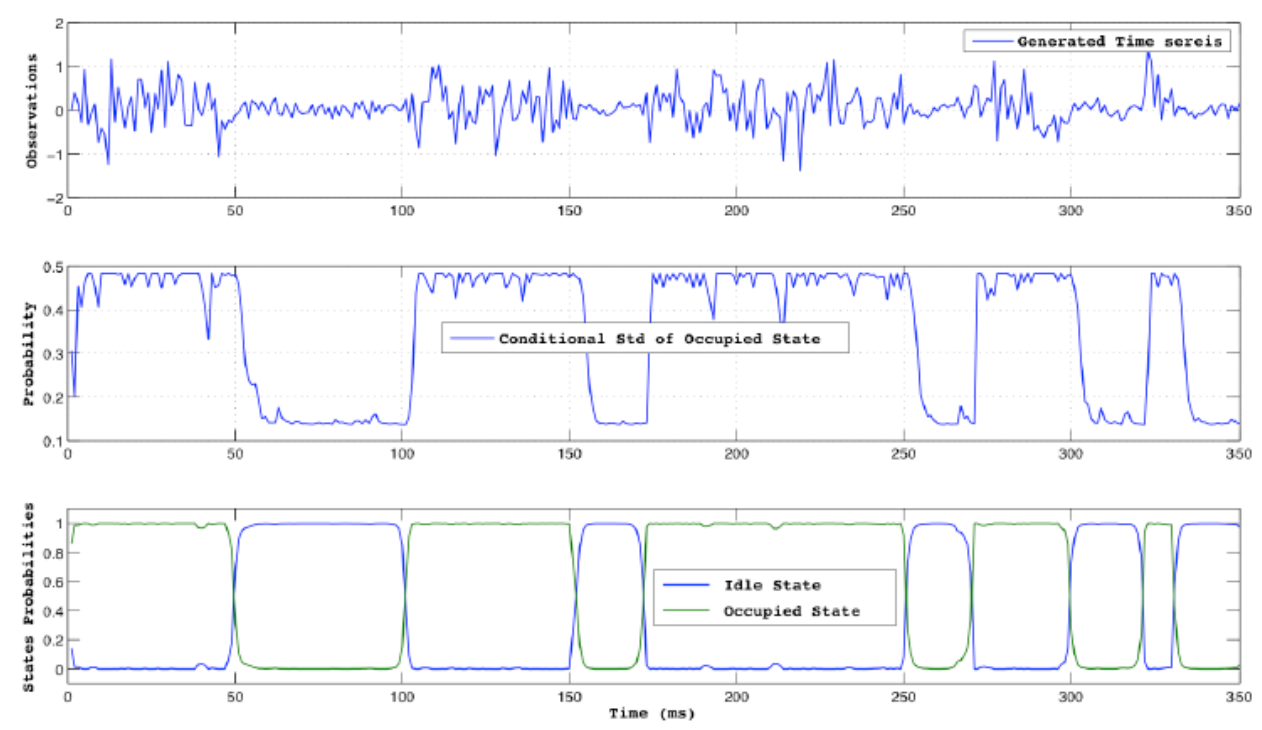

Fig. 4.Shows the performance of the Markov switching model in predicting the series that capture the PU channel state detection sequence

\section{SIMULATION RESULTS}

Fig. 4 shows the performance of the Markov switching model in predicting the time series that generated to capture the detection sequence as in Fig. 3 which, illustrate the detection sequence of single user cognitive radio network with a randomly distributed channel occupancy over time $T=$ $350 \mathrm{~ms}$.

Fig .4 contains three graphs; the top graph shows the generated time series with the following distribution $z_{t} \sim(0.1,0.5)$ for idle state and $z_{t} \sim(0.01,0.2)$ for occupied state " note that choosing the wrong values of $\mu_{z 0}, \sigma_{z 0}{ }^{2}, \mu_{z 1}$ and ${\sigma_{z 1}}^{2}$ may lead to the lack in the performance of the prediction algorithm, so that these values must be chosen with taking equation (3) into account ". The middle graph shows the variation of occupied state prediction probabilities over the time and the bottom graph shows the performance of prediction algorithm and explains the switching process of the PU channel states between idle and occupied.

\section{CONCLUSION}

In this study, we consider the problem of spectrum occupancy status prediction for single user CR network based on Markov switching model. First, we modeled the PU detection sequence as switching time series; we then derived the Markov switching model for it and estimated its parameters using maximum likelihood estimation. Finally we validated our algorithm using simulated testing detection sequence and it shown that the new approach performs very well for predicting the PU channel occupancy status.

\section{ACKNOWLEDGMENT}

This work was supported by the Natural Science Foundation of Jilin Province- China under Grant No. 201215133, and the Sci-tech Development Project of Jilin Province of China under Grant No. 20130521015JH. 


\section{REFERENCES}

[1] Clancy, T., \& Walker, B. (2006, November). Predictive dynamic spectrum access. In Proc. SDR Forum Technical Conference.

[2] Chen, Z., \& Qiu, R. C. (2010, March). Prediction of channel state for cognitive radio using higher-order hidden Markov model. In IEEE SoutheastCon 2010 (SoutheastCon), Proceedings of the (pp. 276-282). IEEE

[3] Chen, Z., Guo, N., Hu, Z., \& Qiu, R. C. (2011, March). Channel state prediction in cognitive radio, Part II: Singleuser prediction. In Proceedings of IEEE SoutheastCon ( $p p$. 50-54)

[4] Chen, Z., Guo, N., Hu, Z., \& Qiu, R. C. (2011). Experimental validation of channel state prediction considering delays in practical cognitive radio. In Vehicular Technology, IEEE Transactions on, 60(4), 13141325.
[5] Frühwirth-Schnatter, S. (2006). Finite Mixture and Markov Switching Models: Modeling and Applications to Random Processes. Springer.

[6] Hamilton, J. D. (1994). Time series analysis (Vol. 2). Princeton: Princeton university press.

[7] Kuan, C. M. (2002). Lecture on the Markov switching model. Institute of Economics Academia Sinica. at homepage.ntu.edu.tw/ ckuan/pdf/Lec-Markov_note.pdf

[8] Kim, C. J. (1994). Dynamic linear models with Markovswitching. Journal of Econometrics, 60(1), 1-22 .

[9] Park, C. H., Kim, S. W., Lim, S. M., \& Song, M. S. (2007, December). HMM based channel status predictor for cognitive radio. In Microwave Conference, 2007. APMC 2007. Asia-Pacific (pp. 1-4). IEEE

[10]Yarkan, S., \& Arslan, H. (2007, September). Binary time series approach to spectrum prediction for cognitive radio. In Vehicular Technology Conference, 2007. VTC-2007 Fall. 2007 IEEE 66th (pp. 1563-1567). IEEE 